\title{
Research on Industrial Control Strategies of Industrial Ethernet and Fieldbus
}

\author{
Xuefeng $\operatorname{Tan}^{1}$, Yachun Dong ${ }^{2}$ \\ ${ }^{1}$ Department of Automation, Jilin Vocational College of Industry and Technology, Jilin, 132013, \\ China \\ ${ }^{2}$ Electric workshop, PetroChina Jilin Petrochemical Co ethylene glycol plant, Jilin, 132022, China \\ xuefeng_tan@yeah.net
}

Keywords: Industrial Ethernet, Fieldbus, Network Integration

\begin{abstract}
This paper designs a system structure scheme of industrial Ethernet integrating fieldbus, and gives application examples of MODBUS

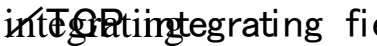

fieldbus. Meanwhile, according with requirement of power equipment fault diagnostics system, this paper gives a design scheme of embedded data acquisition and fault diagnostics system based on industrial Ethernet and fieldbus, also with application examples.
\end{abstract}

\section{Introduction}

Industrial control network is a newly developed network technology in the field of automatic control. It is a combination of computer networks, communications and automation technology. With the development of automation, computers, telecommunication, networks and other technology, enterprise information integration system is rapidly growing. This system will cover everything from local control to various levels of monitoring, marketing, management, and each link of procurement of raw materials, production and processing, and supply chain processes stretch to the finished products storage, transportation, and sales and markets all over the world to suit application requirements of the integrated management and control [1-3]. Enterprise information system development puts forward higher requirements for industrial data communication openness, the functionality and performance to control the bottom network.

There are still some problems in Industrial control network applications fieldbus and industrial Ethernet, the main features of fieldbus is the saving of installation costs and maintenance expenses, In terms of hardware, the price is not lower than a traditional DCS or PLC, even slightly higher than them. However, in China, on the one hand users are very sensitive to the price of the hardware, while imports fieldbus hardware price is very high. Relatively speaking, the domestic price of DCS or PLC system is relatively low. On the other hand, domestic labor costs low, so the design, management, installation and commissioning cost of the project are lower than abroad [4-6]. It's also an important reason that not much fieldbus used in the domestic application. Despite the development of fieldbus for many years, in the economic, security and other aspects of the existing DCS or PLC systems compared to no obvious advantage, so the current application of fieldbus systems focused on the transformation of a small system and the old system .This causes two problems: on the one hand, small systems generally do not join management automation and remote diagnostic capabilities, and therefore it cannot play the field bus system to reduce operation and maintenance cost advantages; on the other hand, a large number of problems in the system or critical systems small systems cannot be reflected, fieldbus superiority cannot be fully reflected, which also hindered the development of fieldbus[7-8].

Inevitable future industrial control networks to open, multi-network coexist direction. Different industrial Ethernet systems need to be interconnected, industrial Ethernet systems and field bus systems also need to be interconnected, plus the emerging industrial wireless networks, the degree of complexity far exceeds previous single control network era. In the hybrid network interconnection process, we will certainly be some tricky and unpredictable problems, so careful study is necessary to Industrial Ethernet and fieldbus key issues at the interconnection. 


\section{System architecture design on Industrial Ethernet Fieldbus Integration}

Inevitable future industrial control networks to open, multi-network coexist direction. Different industrial Ethernet systems need to be interconnected, industrial Ethernet systems and field bus systems also need to be interconnected, plus the emerging industrial wireless networks, the degree of complexity far exceeds previous single control network era. In the hybrid network interconnection process, we will certainly be some tricky and unpredictable problems, so careful study is necessary to Industrial Ethernet and fieldbus key issues at the interconnection.

Industrial Ethernet fieldbus systems integration architecture is shown in Figure 1. The architecture is based on the popular embedded platform, the main use of the ARM and x86 embedded systems hardware platform, the embedded hardware platform to study the MODBUS and other industrial Ethernet and fieldbus technology.

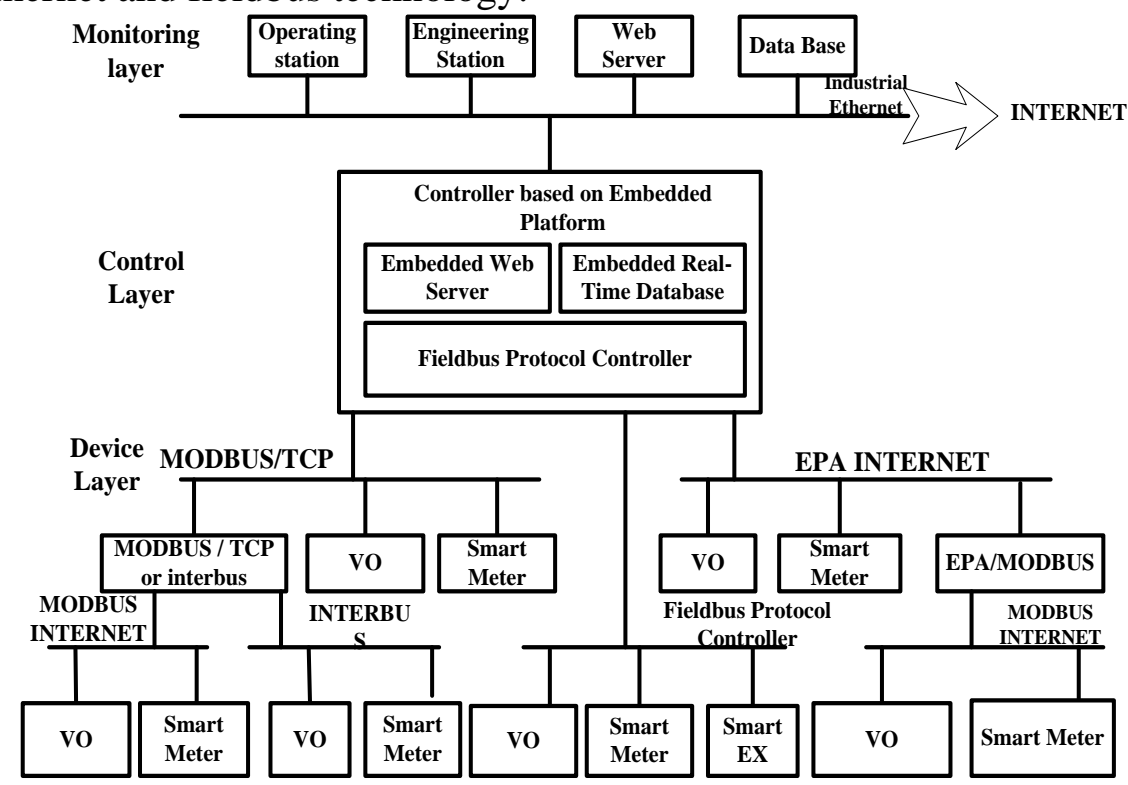

Figure 1. Embedded Platforms Based Industrial Ethernet Integrated Fieldbus Architecture Design

The platform is divided into three layers. Device layer supported by the Industrial Ethernet or fieldbus intelligent I/O devices. Currently this intelligent I/O devices have been very popular, many applications in the industrial field. Between the devices through different layers of different network protocol conversion gateway implementations, such equipment is also relatively popular, and it's also can be developed on one's own..

Control layer consists of a controller based on embedded platform. The controller via Industrial Ethernet fieldbus communication with field devices or via Industrial Ethernet devices to communicate with the monitoring level, mainly for data acquisition, data processing, condition monitoring and network communications, and other functions.

Monitoring level by the operator station, engineer station, Web servers and database servers and other components, mainly to complete the operation and monitoring of field devices, data storage, Web publishing, and other functions.

The core of the architecture is based on the embedded level controller, which provide external data acquisition interface, network communication interfaces and human-computer interaction interfaces. Meanwhile, the controller also integrates an embedded Web server and embedded real-time database. By porting and modifying sophisticated embedded Web server to the target platform, supporting CGI, JSP, ASP and other scripting languages, depending on the application independently achieve the desired Web service. The key is to build an embedded real-time database to establish a data model, which determines the data to be accessed and manipulated way, application performance and feasibility.

The main features of this architecture:

First, the integration of a variety of mixed networks, both directly support industrial Ethernet and fieldbus network, also supports integrated Industrial Ethernet fieldbus hybrid network. According to 
the actual situation of the industrial site, the option of using one or several networks, increase the flexibility of on-site use.

Second, the field devices that support industrial Ethernet or fieldbus intelligent I/O devices, and strengthen the network communication and diagnostic capabilities of field devices.

Third, the embedded controller provides embedded Web server that provides Web services-bit database server up, engineer station, operator station, easy for device monitor computer monitor through a browser, parameter settings and data communications. .

Fourth, the monitoring layer provides a Web server, remote monitoring of field devices via Interact.

\section{Applications}

Power equipment embedded data acquisition and fault diagnosis platform are taken as an example on Industrial Ethernet fieldbus integration implementation, Aiming at the power of large turbine generator equipment, the platform achieves the main and auxiliary equipment for power plant data acquisition and fault diagnosis.

The overall system architecture. The system is constructed in fieldbus and industrial Ethernet and Interact based on applications. Automation technology, computer technology, network communication technology, embedded technology and Internet technology are extensively used.

The overall architecture of the system is shown in Figure 2.

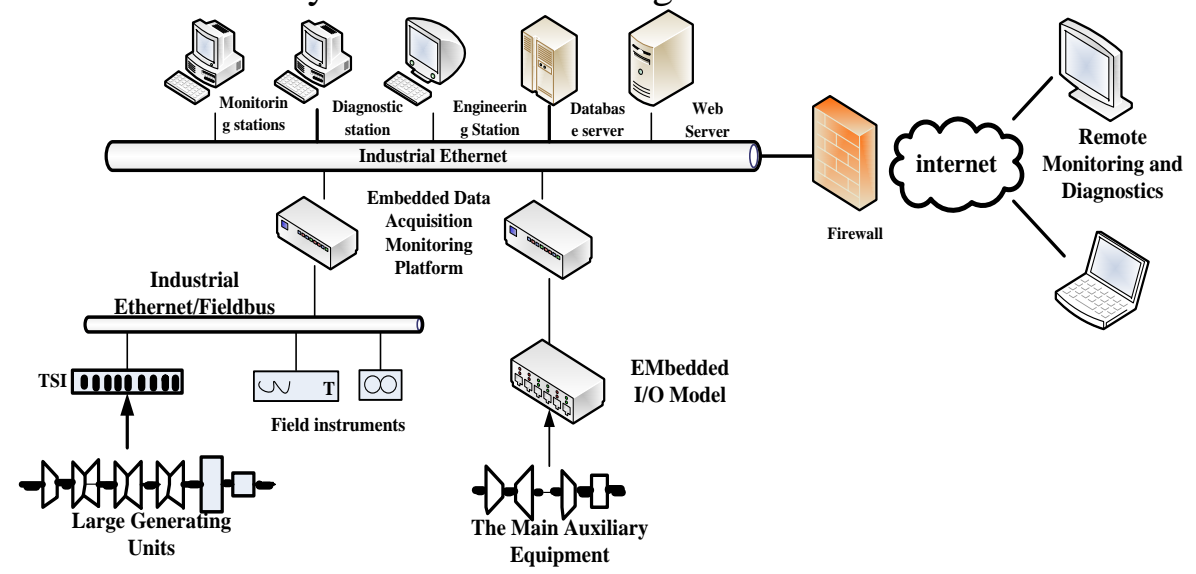

Figure 2. Power Equipment embedded data acquisition and fault diagnosis platform amnesty overall chart

The system mainly includes three parts:

(1)Embedded data acquisition monitoring platform from generators TSI system or various sensing din unit in a variety of operational data collected online. Such as vibration, displacement, differential expansion, a key phase, speed, pressure, temperature, and various process parameters. The platform can support a variety of serial communications, industrial Ethernet, fieldbus, such as data collection. After collecting data through a variety of signal analysis and processing techniques such as FFR transform, wavelet transform preprocessing techniques to be sent via the LAN monitoring and diagnostic center, fault diagnosis expert system platform through the formation of the various features of the analysis process map for local monitoring and diagnostic workstations, remote users, and experts and monitoring real-time monitoring and state analysis.

(2) Monitoring and diagnostic centers mainly by guillotine fault diagnosis expert system, Web server, database server, monitoring and diagnostic workstations and other components. It is through the embedded data acquisition monitoring platform for real-time network communication and access to real-time data stored in wells real-time database. In the real-time database platform troubleshooting techniques such as the use of modern vector machines, neural networks and expert systems to support the formation of fault diagnosis expert system, intelligent fault diagnosis of the typical characteristics of generator sets, while diagnostic results released by the Web server to the remote diagnostic platform for remote diagnosis expert analysis. 
(3) Remote diagnosis expert system and Interact experts and get real-time monitoring of user data from the central server for real-time remote monitoring voltage, and connect with the database on the server to get the status of historical data analysis. Meanwhile, the central server through intelligent diagnostic results released, combining diagnostic expert analysis advice for the unit to implement condition-based maintenance planning and decision support to provide guiding repair comments.

MODBUS/TCP industrial Ethernet and MODBUS fieldbus can achieve seamless integration, but MODBUS/TCP uses the TCP port packet checksum mechanism instead of the MODBUS of CRC or LRC check mechanism. The integrated network monitoring system of MODBUS/TCP and MODBUS can be divided into two levels including monitor layer and the device layer, a communication network composed of two parts including MODBUS fieldbus network and MODBUS/TCP industrial Ethernet, which are connected by the network protocol conversion gateways. System structure is shown in Figure 3.In the Figure engineer stations, operating stations monitor the entire network from a PC computer as the control layer. MODBUS/TCP protocol conversion gateways and field devices implemented by embedded systems.

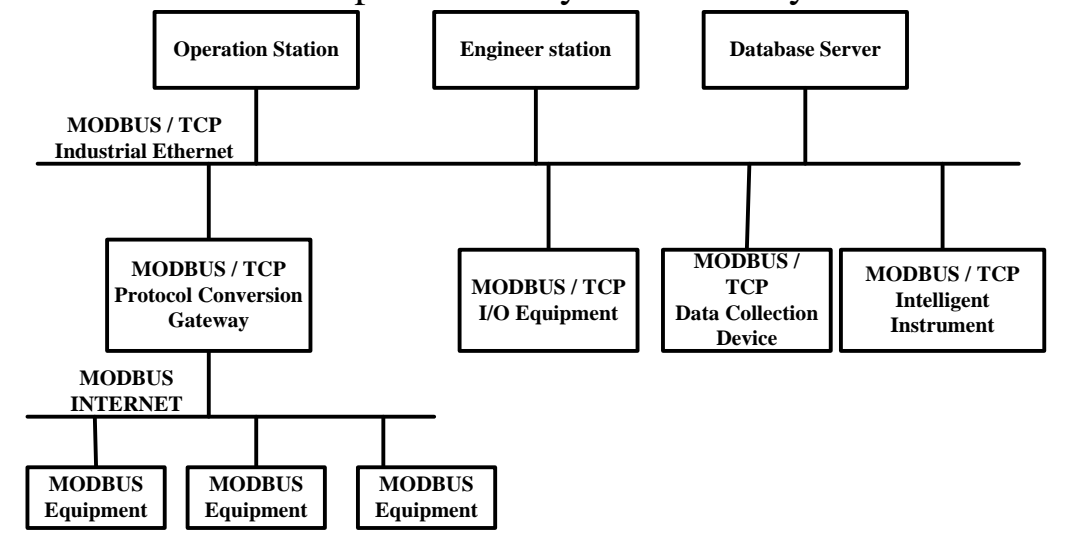

Figure 3. MODBUS/TCP and MODBUS hybrid network structure plan

As shown above, hybrid network has device node supporting MODBUS/TCP protocol data, and MODBUS and MODBUS/TCP protocol conversion gateways.

Supports MODBUS/TCP protocol data acquisition node includes signal acquisition, data processing and data transmission, in some cases acquisition node also need to have the data display.

Embedded data acquisition system uses Philips's main chip LPC2220 as microcontroller hardware design. LPC2220 microcontroller is based on the real-time emulation and embedded trace 32 ARMTRDMI-S cores, 144-pin package, low power consumption, having many 32 timers, 8-channel 10-bit ADC, PWM outputs, and nine external interrupt pin. It is suitable for industrial control, medical systems, access control, and electronic cash registers (POS) and so on. By configuring the bus, it provides up to 76 GPIO.

Hardware structure schematic diagram of the data acquisition system shown in Figure 4, the core chip are LPC2220, MAXI25, the rest also includes must-be-stored module, clock module, display module and the serial module. Analog signal acquisition portion of the embedded data acquisition system uses MAXIM's MAXI25 chip to complete. MAXl2 is a high speed, multi-channel, 14-bit analog-to-digital conversion chip. The chip has a 14 conversion time of 3 us of successive approximation analog-to-digital conversion circuit, 4X 14-bit RAM, DSP or ARM bus can be connected to three apricot output devices, as well as four-chip sample and hold, each sample and hold input corresponds to a 2-to-1 analog input (a total of eight analog input channels, four one group, divided into $\mathrm{A}$ and $\mathrm{B}$ groups), output by 4 to 1 switch to the $\mathrm{A} / \mathrm{D}$ converter. 


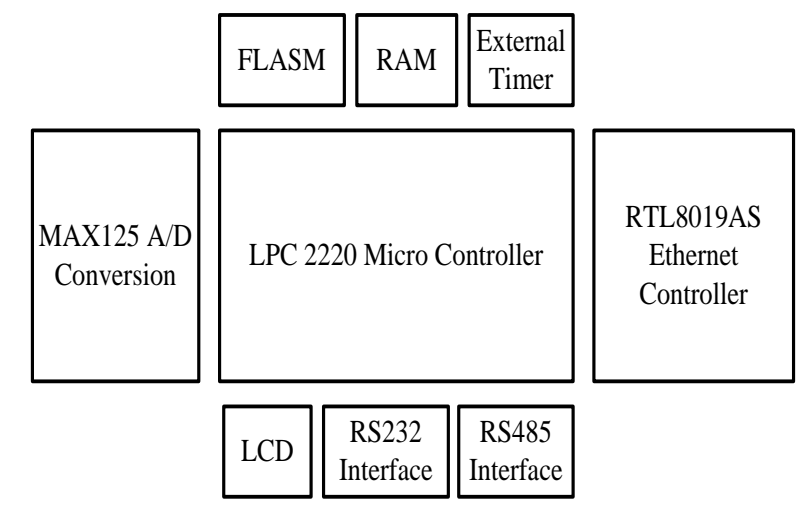

Figure 4. Schematic diagram of the data acquisition system hardware

MAXl25 can be collected by the switching of the switch through eight channels, resulting in the digital conversion which is stored in the RAM $4 \times 14$. Timing characteristics of its data access and parallel interface bus release is compatible with the characteristics of ARM7 series chip bus, so the two can be connected directly without wait states. Because MAXl25 supply power to $+5 \mathrm{~V}$, when connected to the LPC2220 with 3.3V power supply need to use the bus level converter chip 74LVC245A, which uses the bus to read and write control signals change direction, automatically apply external LPC2220 interrupt EINT0 after the conversion is completed, read A / D data will automatically undo the break. In addition, the forward channel needs to increase access limiting protection, voltage follower and filter input circuit.

\section{Conclusion}

Proposed an integrated Industrial Ethernet fieldbus embedded system architecture design program, which uses a hybrid network approach of industrial Ethernet and fieldbus, for many industrial applications, and designed a variety of integrated Industrial Ethernet fieldbus applications, as well as applications to directly use of industrial Ethernet or fieldbus.

\section{Reference}

[1] F.Dopatka, R. Wismüller, “Achieving realtime capabilities in Ethernet networks by edge-colorin g of communication conflict-multigraphs”, inProc. of the IASTED Internat. Conf. on Parallel and D istributed Computing and Networks 2006, Innsbruck, Acta Press, Zurich, pp. 180-185, Feb. 2006. [2] M. Felser, ”Real-Time Ethernet - industry prospective“. In: Proc. of the IEEE, Vol 93, No. 6, June 2005, pp. 1118-1128.

[3] I. Holyer, “The NP-completeness of edge-coloring”, in: SIAM Journal on Computing 10(4), Phil adelphia, PA, 1981, pp.718-720.

[4] G. Schnell, "Subsystem in der Automatisierungs- und Prozesstechnik“ (in German), 5th revised and extended edition, Braunschweig, Vieweg, 2003.

[5] M. Popp, "Das Profinet IO-Buch - Grundlagen und Tipps für Anwender“ (in german), published by Hüthig, Heidelberg, 2005.

[6] F. Dopatka, R. Wismüller, “A top-down approach for real-time industrial-Ethernet networks usi ng edge-coloring of conflict-autographs”, in Proc. of the Internet. Symp. On Power Electronics, Ele ctrical Drives, Automation and Motion (SPEEDAM), Taormina, IEEE Explore, pp. 883-890, May 2 006.

[7] Rockwell Automation, “Vertical Integration: Mitt Eminem ensign Feldbusprotokoll vow Interne t sum Sensor"(in German),

[8] D. Marx, "Graph coloring problems and their applications in scheduling”, John von Neumann P hD students Conference, Budapest University of Technology and Economics, 2003. 